
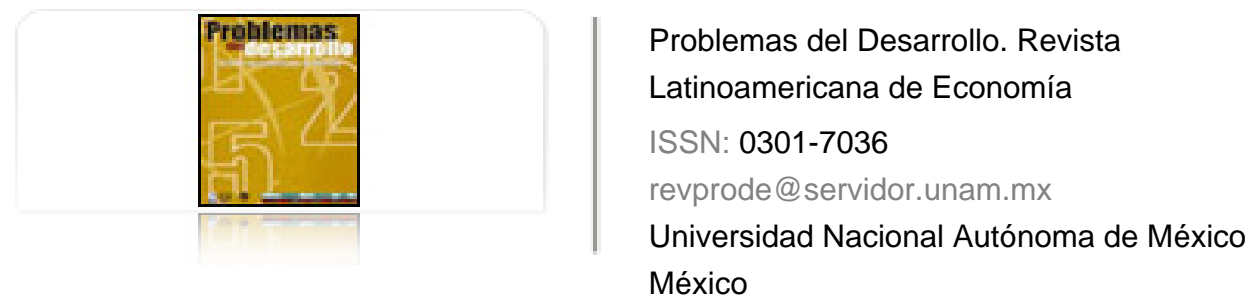

Martínez González, Blanca Gloria

Plusvalor, ingreso de trabajadores autónomos y diferencias nacionales de tasas de plusvalor Problemas del Desarrollo. Revista Latinoamericana de Economía, vol. 36, núm. 142, julio-septiembre, 2005, pp. 101-125

Universidad Nacional Autónoma de México

Distrito Federal, México

Disponible en: http://www.redalyc.org/articulo.oa?id=11820079007

Cómo citar el artículo

- Número completo

- Más información del artículo

Página de la revista en redalyc.org

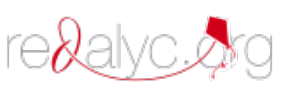

Sistema de Información Científica

Red de Revistas Científicas de América Latina, el Caribe, España y Portugal Proyecto académico sin fines de lucro, desarrollado bajo la iniciativa de acceso abierto 


\section{PlusValor, INGRESO de tRABAJAdORES AUTÓNOMOS Y DIFERENCIAS NACIONALES DE TASAS DE PLUSVALOR}

\section{Blanca Gloria Martínez González*}

Fecha de recepción: 31 de marzo de 2005. Fecha de aceptación: 11 de agosto de 2005.

\section{Resumen}

Los países de menor productividad tienen tasas de plusvalor más altas que los de mayor productividad. Este resultado parece contradecir las necesidades de la acumulación: las naciones con más producción lo son porque tienen mayor composición orgánica promedio y precisan, por tanto, de una mayor tasa de plusvalor para compensar esa elevada composición orgánica de capital. En este artículo se examina qué han hecho distintos autores con los ingresos de los trabajadores autónomos en sus estimaciones de tasa de plusvalor; se ilustra, con el caso de México, cómo se pueden modificar las estimaciones de tasa de plusvalor y las conclusiones sobre diferencias de tasa de plusvalor entre paises con diferencias significativas de productividad y se plantean algunas de las complicaciones teóricas del análisis de la relación entre el trabajo autónomo y tasa de plusvalor.

Palabras clave: tasa de plusvalor, trabajo autónomo, productividad, valor, comparación internacional

\section{Abstract}

Countries with lower productivity have rates of surplus value that are higher than those with greater productivity. This result seems to contradict the need for accumulation: countries with higher productivity are like that because they have greater average organic composition and precisely for that reason a higher rate of surplus value to compensate for their elevated organic composition of capital. In this article we examine what several writers have used in their estimates of the rate of surplus value, i.e. the incomes of autonomous workers. Using Mexico as a case study, it is shown how their estimates of surplus value can be modified and their conclusions on differences in the rate of surplus value between countries with significant differences in productivity. Some of the theoretical complications of the analysis are presented on the relation between autonomous labor and the rate of surplus value.

Keywords: rate of surplus value, autonomous work, productivity, value, international comparison

* Doctorante en ciencias económicas de la UAM. La autora agradece los valiosos comentarios de los re visores anónimos del artículo, así como los de Alejandro Valle Baeza, aunque los exculpa de los errores que permanezcan en el trabajo.Correo electróncio: gloria@servidor.unam.mx 


\section{Résumé}

Les pays dont la productivité est moindre ont des taux de plus-value plus élevés que ceux dont la productivité est plus grande. Ce résultat semble contredire les besoins d'accumulation: les nations qui ont une production plus importante ont une composition organique moyenne plus grande et précisent, pour autant, d'un taux de plus-value plus grand pour compenser cette composition organique de capital élevée. Dans cet article, on examine ce qu'ont fait différents auteurs avec les revenus des travailleurs autonomes dans leurs estimations du taux de plusvalue; on illustre, grâce à avec l'exemple du Mexique comment on peut modifier les estimations du taux de plus-value et les conclusions sur différents taux de plus-value entre les pays avec des différences significatives de productivité et on présente quelques complications théoriques de l'analyse de la relation entre le travail autonome et le taux de plus-value.

Mots clés: taux de plus-value, travail autonome, productivité, valeur, comparaison internationale.

\section{Resumo}

Os paises de produtividade menor têm taxas de mais-valia mais elevadas do que aqueles de produtividade maior. Este resultado parece contradizer as necessidades da acumulação: as nações com maior produção são assim porque têm maior composição orgânica promédio e precisam, como consequência, duma taxa de mais-valia mais elevada para compensar a elevada composição orgânica de capital. Neste artigo estuda-se o que diferentes autores têm feito com os ingressos dos trabalhadores autônomos nas suas estimações de taxa de maisvalia; exemplifica-se com o caso do México como é que podem-se alterar as estimações de taxa de mais-valia e as conclusões sobre as diferenças de taxa de mais-valia entre paises com diferenças significativas de produtividade e sugerem-se algumas das complicações teóricas do análise da relação entre o trabalho autônomo e taxa de mais-valia.

Palavras chave: taxa de mais-valia, trabalho autônomo, produtividade, valor, comparação internacional. 


\section{Introducción}

$\mathrm{E}$

n otro trabajo acerca de la tasa de plusvalor, Martínez (1999) encontró que los nes de mayor productividad lo son porque tienen mayor composición orgánica promedio y precisan, por tanto, de una mayor tasa de plusvalor para compensar esa elevada composición orgánica del capital. Parece, entonces, que en los países capitalistas atrasados hay una superexplotación, mencionada por algunos investigadores. Los hallazgos de Martínez (op. cit.) adolecían de diversos problemas, uno de los más relevantes era el que aquí se abordará: la relación entre tasa de plusvalor y trabajo autónomo o cuenta propia. En el Panorama social de América Latina (CEPAL, 2003), se aprecia la importancia de ese tipo de trabajo en el continente y eso seguramente ilustra la relevancia del problema en el mundo subdesarrollado: entre $20 \%$ y $40 \%$ del empleo corresponde a trabajadores por cuenta propia y de ellos, sólo una pequeña porción corresponde a profesionales y técnicos. Los ingresos de esos trabajadores se incluyen en la contabilidad social junto con las ganancias capitalistas. ¿No es eso la explicación de la mayor tasa de plusvalor de los países atrasados?

El sector de autoempleo en Japón es grande comparado con el de Estados Unidos. Durante el periodo 1958-60, por ejemplo, nuestras estimaciones revelan que en Japón, los trabajadores autoempleados constituían aproximadamente $30 \%$ de la fuerza de trabajo (excluyendo a los trabajadores familiares no pagados), comparado con el 14\% en los Estados Unidos. Más tarde en el período 1977-80, eran el 18 por ciento y el 9 por ciento de las fuerzas de trabajo respectivas (Kalmans, 1992:98, nota).

En el presente trabajo se verá que la tasa de plusvalor ajustada mediante la exclusión del ingreso de los trabajadores autónomos de los excedentes de operación contrasta dramáticamente con la estimada por Martínez (1999). Se mostrará que, no obstante lo anterior, los nuevos resultados apoyan el hallazgo según el cual la tasa de plusvalor es mayor en México que en países desarrollados.

Aquí se señala la relevancia del ingreso de los trabajadores autónomos en la estimación de la tasa de plusvalor; se ilustra, con el caso de México, cómo se pueden modificar las estimaciones de dicha tasa y las conclusiones acerca de las diferencias de ésta en países con diferencias significativas de productividad y se plantean algunas de las complicaciones teóricas que surgen de tal análisis.

En el primer apartado se aborda el problema empíricamente; se revisa qué hacen otros autores con el ingreso de los trabajadores autónomos en sus estimaciones de la tasa de

\section{Desaarrollo}


plusvalor en otros países, se emiten algunas observaciones críticas y se expone un ajuste propio de esta tasa en el caso específico de México. En el segundo apartado se examinan las razones teóricas que se explicitan o se infieren del tratamiento que los autores referidos hacen de los ingresos de trabajadores autónomos. También se enfatizan las dificultades teóricas que introduce dicho trabajo en el cálculo de la tasa de plusvalor. Finalmente, se plantean las conclusiones del trabajo, entre las cuales se destaca que los nuevos resultados apoyan los hallazgos originales de Martínez (1999), según los cuales la tasa de plusvalor en México es mayor que la de países desarrollados y que en el tratamiento empírico del ingreso de los trabajadores autónomos predomina un enfoque inadecuado, pues se trata a este tipo de ingresos como plusvalor o capital variable.

\section{Ingresos de trabajadores autónomos en estimaciones de tasa de plusvalor}

\section{Ubicación del problema}

La teoría del plusvalor es, sin duda, junto con la teoría del valor, uno de los aspectos más polémicos del trabajo de Marx y de sus seguidores. Recuérdese que Marx encontraba en los conceptos de trabajo abstracto y de plusvalor sus dos aportaciones más importantes y las mayores críticas se dirigen hacia las partes de su teoría en las cuales se tratan estas cuestiones.

La teoría del plusvalor es el marco en el cual se mueve la presente investigación. Si se recuerda, su estudio fue abordado en muchas porciones de la obra de Marx, por ejemplo, en sus teorías sobre la plusvalía (Marx y Engels, 1987). En sus estudios, aparecen como problemas centrales de la teoría del plusvalor el origen y las leyes del excedente capitalista; además, criticó las concepciones de los economistas clásicos, especialmente de Adam Smith (1958) y David Ricardo (1959).

Los marxistas posteriores discutieron problemas adicionales a los planteados por Marx, muchas veces movidos por la importancia política del tema. Así, Rosa Luxemburgo (1967), Lenin (1974, v.3) y Bujarin (1975), entre otros, debatieron los problemas de realización como un aspecto central para entender el capitalismo de su época.

Para las naciones atrasadas ha resultado muy relevante la discusión acerca del plusvalor transferido a los países centrales. Emmanuel (1972), Samir Amin (1975) y Ruy Mauro Marini (1989), por ejemplo, discuten acerca del intercambio desigual, de la transferencia de valor y de la dependencia de una economía; el último desarrolla un concepto de superexplotación en las naciones atrasadas. Ernest Mandel (1972) y Bettelheim (1972) polemizan sobre las diferencias de productividad y de salarios entre países.

Más particularmente, el estado actual del desarrollo de la teoría del plusvalor se puede dividir en tres grandes líneas de investigación: $i$ ) medición de la tasa de plusvalor, la cual 
destaca la aportación de Shaikh y Tonnak (1994); ii) contrastación empírica de la teoría de la tasa de plusvalor en un país, y iii) comparaciones internacionales de dicha tasa. El desarrollo habido en las dos primeras líneas ha sido determinante en los avances de las comparaciones internacionales. En la línea de la contrastación empírica de la teoría sobre la tasa de plusvalor en un país, se encuentran dos tipos de trabajos: 1) los que corroboran la inclinación creciente de la tasa planteada por la teoría y 2) los que confirman tanto esa tendencia como las relaciones entre la tasa de plusvalor y otras categorías, además de la composición orgánica de capital y la tasa de ganancia, previstas por la teoría. Trabajos como los de Cockshott et al. (1995), Moseley (1991) y Smith Murray (1993), por ejemplo, encuentran un fuerte soporte empírico de las tendencias propuestas por la teoría: a) decreciente de la tasa de ganancia, $b$ ) creciente de la tasa de plusvalor, y $c$ ) creciente de la composición orgánica del capital. En la línea de las comparaciones entre países son destacables los trabajos de Amsden (1981), Izumi (inédito), Kalmans (1992), Guerrero y Díaz (1999) y Martínez (1999b y 1996).

En Martínez (1999) importaba de manera central analizar la productividad, relacionada con el grado de desarrollo de las fuerzas productivas, y la tasa de plusvalor. Aquí, el interés es todavía más específico: examinar la relación entre los ingresos de los trabajadores autónomos y dicha tasa, así como su efecto en las diferencias de las tasas entre naciones.

El problema del ingreso de los trabajadores autónomos en la estimación de la tasa de plusvalor es diferente según se trate de países subdesarrollados o desarrollados. En general, en ambos, los ingresos de los trabajadores autónomos se han venido contabilizando en los excedentes de operación de las cuentas nacionales.

En las naciones subdesarrolladas — hasta antes de los noventa, en algunos y hasta la fecha en otros-, no ha sido posible separarlos de otros ingresos identificables con las ganancias capitalistas. En el caso de México, ajustar dichos excedentes mediante la exclusión del ingreso de los trabajadores autónomos para estimar la tasa de plusvalor conduce a otros problemas prácticos, como se verá más adelante. En los países desarrollados, este tipo de ingresos también está contabilizado en los excedentes de operación, pero se puede identificar. Diversos autores distribuyen el monto de estos ingresos entre las formas monetarias de capital variable y plusvalor. No se ha encontrado que tales autores expliciten sus razones teóricas. Parece haber más bien consideraciones prácticas cuando lo hacen así, pues lo que pretenden es no tratar este tipo de ingresos en su totalidad como ganancias capitalistas.

Esta investigación se considera que de ingresos de los trabajadores por cuenta propia hay que distinguir, en principio, dos tipos: a) los asalariados disfrazados de autónomos, trabajadores subcontratados por capitalistas que, por esa circunstancia, son en realidad asalariados, y b) los autónomos puros, es decir, trabajadores auténticamente no asalariados. El plusvalor generado por los primeros aparece en el valor agregado de las empresas

\section{Desaarrollo}


capitalistas que los subcontratan, mientras que el excedente capitalista producido en unos casos o apropiado en otros por los segundos, habría que calcularlo con base en la diferencia entre su tasa de ingreso y la expresión dineraria del valor de la economía. Sobre esto se volverá más adelante y se tratará con más amplitud.

En la literatura previa a esta investigación no subyace tal razonamiento a los tratamientos empíricos. Éstos son de tres tipos: $a$ ) el predominante, bajo el cual se divide el valor nuevo que produce un trabajador independiente entre capital variable y plusvalor; $b$ ) el alternativo, de acuerdo con el cual se separa dicho valor nuevo del producido en forma capitalista; y c) un excepcional, con base en el cual todo el ingreso de los trabajadores autónomos se agrega al capital variable.

\section{En estimaciones de tasa de plusvalor de otros autores}

En sus estimaciones empíricas de categorías marxistas en Estados Unidos, Shaikh y Tonnak (1994) calculan el capital variable a partir del valor total de los salarios, considerando la diferencia entre trabajo productivo e improductivo y estimando un equivalente salarial de las personas autoempleadas.

El valor añadido por las propiedades y las sociedades no está dividido entre salarios y beneficios. Ignorar el equivalente salarial de las personas autoempleadas (propietarios y socios) equivale a tratar todo el valor añadido como renta de tipo beneficio. Hemos optado, en cambio, por imputar un equivalente salarial al trabajo de las personas autoempleadas y tratar el resto del valor añadido como renta de tipo beneficio (que puede ser negativa en caso de pérdidas) (Shaikh y Tonak, op. cit.:112, nota).

Kalmans (1992), en su estudio comparativo de la tasa de plusvalor entre Estados Unidos y Japón, y Cronin (2001), en su estimación de categorías económicas clásicas en Nueva Zelanda, proceden análogamente. Kalmans hace una consideración práctica en su estimación basada en insumo-producto: en las matrices el ingreso de los trabajadores autoempleados aparece como componente del excedente de operación, de manera que si éstos se ignoran, su ingreso aparece en el plusvalor y se infla la tasa de esta categoría:

Sostenemos, para ambos propósitos teóricos y prácticos que tiene más sentido tratar a los campesinos autoempleados, a los autoempleados propietarios de pequeñas empresas industriales familiares como trabajadores productivos en lugar de trabajadores improductivos implícitamente, y contabilizar su salario equivalente como una parte constitutiva de capital variable, en lugar de componente del plusvalor (Kalmans, 1992:71).

Cockshott et al. (1996), aunque incluyen los ingresos de los trabajadores autónomos en el plusvalor, en sus estimaciones de categorías marxistas en Reino Unido dicen: “Aceptamos el argumento de Maniatis de que en principio es posible dividir la renta del autoempleo entre una porción autogenerada, equivalente al valor creado por una persona en un año de trabajo, y una porción que representa la renta de explotar a otros" (op. cit.:59). 
Gouverneur (1990) utiliza el mismo método: divide la renta de la producción mercantil no capitalista entre capital variable y plusvalor, pues imputa un equivalente salarial a los trabajadores independientes y lo suma al capital variable para calcular la tasa de plusvalor.

Sharpe (1982) opina igual, pero procede distinto: "He decidido asignar toda la renta de los negocios no constituidos en sociedad anónima a la renta bruta del trabajo" (op. cit.:398). $\mathrm{Al}$ parecer, lo hace así porque piensa que el ingreso de los trabajadores autónomos iguala al de los asalariados, debido a las rentas relativamente bajas de los negocios no constituidos en sociedades anónimas en el sector agrícola y del pequeño comercio. Ésta no es una razón válida, pues que dichos ingresos sean cuantitativamente semejantes no los hace iguales en términos cualitativos. Acerca de las cualidades del trabajo autónomo se tratará en el siguiente apartado.

Mateo Tomé (2003) estima tres tasas de plusvalor en Venezuela. En dos de ellas excluye el ingreso de los trabajadores autónomos del valor agregado, en una de las cuales distingue los ingresos de trabajadores productivos de los de trabajadores improductivos, excluye los salarios de éstos del capital variable y los agrega al plusvalor, y en la otra mantiene dichos salarios junto al capital variable. En un tercer cálculo, procede como Sharpe: ajusta la tasa de plusvalor mediante la adición del ingreso de los autónomos al capital variable.

Mage (1963), para estimar el plusvalor en la economía estadounidense, intentó restar al valor agregado el ingreso del trabajo productivo y después el del trabajo autónomo, con base en la media anual de los ingresos de los trabajadores de tiempo completo en cada industria. A diferencia de los autores antes referidos, no añade este último a su estimación monetaria del capital variable. Así, esa parte de los ingresos de autónomos queda fuera del análisis y el resto se entiende que permanece en el plusvalor. ${ }^{1}$

Diego Guerrero (1989), en su trabajo sobre distribución de la renta en España y Moseley (1982) acerca de la tasa de plusvalor en Estados Unidos, consideran que es necesario descontar el ingreso de los trabajadores autónomos del valor nuevo. Moseley (1991) resta al producto neto del sector mercantil el ingreso de los propietarios autoempleados. Supone que éste se puede estimar mediante la participación de las horas trabajadas por los autoempleados en el total de las horas trabajadas, cosa que Shaikh y Tonnak le critican:

Moseley también resta la renta de las empresas no constituidas (en sociedad) en la agricultura, construcción y servicios, presumiblemente basándose en que representan flujos no capitalistas. Esto nos parece improbable. Hasta la invención de la figura legal llamada

"Mage comete dos errores en este procedimiento. Primero, al deducir la parte productiva de las rentas del sector de empresas no constituidas en sociedad anónima, descuenta simplemente las rentas de los 'agricultores, intermediarios financieros y médicos privados' (Mage, 1963:65), algo incoherente con su definición de trabajo productivo, pues no descuenta la actividad comercial, por ejemplo. En segundo lugar, aunque resta esta porción del cálculo de la plusvalía, no añade estos salarios imputados al cálculo del capital variable" (Cámara, 2003:215, nota).

\section{Desaarrollo}


'sociedad' todas las empresas capitalistas no estaban constituidas, por lo que la forma no constituida de estas empresas particulares no las convierte en no capitalistas. Ni hay una razón que obligue a creer que la mayor parte de la agricultura, la construcción y los servicios sea no lucrativa (1994:185-186).

Es decir — siguiendo a Cámara (2003)—, Moseley sustrae parte del valor nuevo de la producción capitalista y lo suma al valor nuevo de la producción no capitalista. A diferencia, Guerrero (1989) discute las limitaciones de las estadísticas oficiales para separar el valor nuevo que llama capitalista del no capitalista. ${ }^{2}$ Guerrero (2005) señala que la contabilidad nacional no separa los datos de renta entre las categorías sociales de asalariados, capitalistas e intermedios que coexisten en el capitalismo, de manera que cabe calcular la renta que corresponde a cada uno de esos tres grupos. Afirma que se requiere hacer una estimación de qué parte del excedente bruto de explotación corresponde a los trabajadores autónomos y a los capitalistas, para lo cual frecuentemente se supone que cada uno de aquéllos obtiene un ingreso promedio igual al salario promedio de los trabajadores por cuenta ajena.

$\mathrm{Si}$ se piensa en los integrantes de las profesiones liberales, como los miembros típicos de esta capa intermedia, uno podría creer que la renta de éstos es bastante mayor que la de los asalariados. Pero se olvida a menudo que los grandes abogados, médicos o notarios que no son asalariados tampoco son autónomos; son (pequeños) capitalistas que emplean en sus bufetes, clínicas y notarías a un número mayor o menor de asalariados (son empleadores en el sentido de la Encuesta de Población Activa y, por tanto, capitalistas). La mayor parte de los verdaderos autónomos, sin embargo, integra sectores como la hostelería o el comercio y, sobre todo, la agricultura, en todos los cuales la renta media está por debajo de la media de la economía. Por consiguiente, no parece tan descabellado suponer que estos trabajadores tienen, como media, una renta equivalente al asalariado medio (Guerrero, 2005:58).

La metodología propuesta por Guerrero (1989) encuentra continuidad en el trabajo de Cámara (op. cit.), quien llama la atención sobre dos cosas: primero, Marx habló de la imposibilidad de aplicar los conceptos de trabajo productivo e improductivo a ese tipo de producción (mercantil no capitalista), antes de su clasificación como trabajo improductivo; segundo, la necesidad de separar la producción mercantil — a su decir — no capitalista de la producción capitalista. Agrega, siguiendo a Gouverneur (1998) que el trabajo mercantil no capitalista es productivo en un sentido amplio, pero improductivo en sentido estricto, es decir, que contribuye a la creación de valor nuevo aunque no a la creación de plusvalor. "La producción mercantil no capitalista no supone una deducción del valor nuevo, sino que es en sí misma una renta. Sin embargo, esta renta no se puede dividir

2 "De hecho, en el epígrafe de propietarios no se incluyen únicamente a los empleados sin asalariados y trabajadores independientes (que corresponden a la producción mercantil no capitalista) sino también a los propietarios de empresas no constituidas en sociedades, que se corresponden con producción capitalista propiamente dicha" (Cámara, op. cit::217). 
entre capital variable y plusvalía, pues no se trata de producción estrictamente capitalista" (Cámara, op. cit.:213).

Según este autor, el valor nuevo agregado en una economía se descompone en capital variable, plusvalor y valor nuevo de la producción mercantil no capitalista y es necesario usar un procedimiento indirecto para estimar el último. ${ }^{3}$

\section{Observaciones críticas}

El enfoque Shaikh-Tonnak y de quienes siguen su metodología es incorrecto. En primer lugar, sin hacer ninguna distinción entre los trabajadores que la contabilidad nacional denomina autoempleados, tratan una parte de los ingresos autónomos como plusvalor y la otra como capital variable. Tal distribución implica que conciben un proceso de explotación en donde no existe. No hay enajenación ni expropiación de trabajo que produzca plusvalor. Ninguna parte de los ingresos autónomos puede ser capital variable, a menos que se trate de trabajadores autónomos subcontratados, de asalariados disfrazados.

En segundo lugar, sólo cuando la tasa de ingreso autónomo es mayor que la expresión dineraria del valor, una parte de dichos ingresos sería plusvalor. Pero ninguno de los autores que adoptan este enfoque discute tales aspectos.

En este trabajo se comparte la postura de Guerrero (1989) y Cámara (2003), de acuerdo con la cual los ingresos de los trabajadores autónomos no deben ser incluidos en el plusvalor ni en el capital variable. Con esta idea se procede a estimar la tasa de plusvalor ajustada en México. También se coincide en en cuanto a lo que las cuentas nacionales clasifican como trabajadores por cuenta propia, se debe identificar a los verdaderos trabajadores autónomos, los empresarios sin asalariados y trabajadores independientes (ESATIS), a los que se refiere Guerrero (1989). Sólo cabría anotar algunas observaciones que también valen para el ajuste propio de la tasa de plusvalor en México y sobre las que se abundará más adelante.

Dentro del conjunto de trabajadores autónomos hay que distinguir dos tipos: a) los asalariados disfrazados de autónomos, trabajadores subcontratados por capitalistas que, por esa circunstancia, son en realidad asalariados; y b) los verdaderos autónomos, es decir, trabajadores auténticamente no asalariados.

El plusvalor generado por los primeros aparece en el valor agregado de las empresas capitalistas que los subcontratan; el ingreso que perciben debe sumarse a las remuneraciones de trabajadores productivos para estimar el capital variable.

3 En el caso de España "el procedimiento concreto consiste en estimar la serie para el periodo 1954 2001 del número de personas que pertenece al colectivo de los 'empresarios sin asalariados y trabajadores independientes' (ESATIS) y asignarles una renta media. De esta forma obtenemos la renta agregada de esta clase social, que se equipara al valor nuevo de la producción mercantil no capitalista" (Cámara, op. cit::511).

\section{Desaarrollo}


Cuando se trata de verdaderos trabajadores autónomos, su masa de ingresos no es siempre equivalente al valor nuevo de la producción mercantil no capitalista (VNpmnc). Cuando compiten con las empresas capitalistas, las cosas son complejas, pues hay que considerar que las empresas producen más o menos plusvalor de acuerdo con su productividad. Puede resultar, entonces, que los autónomos obtengan menos que el salario medio por causa de su baja productividad. En ese caso, surge un excedente que hay que imputar con base en la diferencia entre tasa de ingreso autónomo y la expresión dineraria del valor, y que se debe restar del valor agregado.

Por otra parte, no se comparte la idea de Guerrero (2005:58) según la cual "los grandes abogados, médicos o notarios que no son asalariados tampoco son autónomos sino pequeños capitalistas, que emplean en sus bufetes, clínicas y notarías a un número mayor o menor de asalariados". Se considera que se trata de trabajadores calificados que se apropian de plusvalor en una magnitud que no se explica por el hecho de emplear asalariados ni el valor que se apropian se justifica por su calificación.

¿Cómo diferenciar las posibles situaciones y cómo afectan cada una al cálculo de la tasa de plusvalor? Para estimar dicha tasa en una economía real, parecería no ser suficiente excluir del excedente de operación una masa de ingresos con base en el número de ESATIS y una tasa salarial promedio. Sin embargo, si se tiene una tasa de plusvalor estimada sin considerar el problema del ingreso del trabajo autónomo y otra estimada con la idea de excluir del valor agregado el ingreso de los trabajadores autónomos, se tienen dos aproximaciones a partir de las cuales es posible extraer conclusiones.

Un ajuste de la tasa de plusvalor en México: exclusión de los ingresos de los trabajadores autónomos

Se estimó una tasa de plusvalor ajustada mediante la exclusión de los ingresos de los trabajadores autónomos de los excedentes de operación y la aproximación a las ganancias monetarias. Lo anterior se hizo con base en el cruce de datos del Sistema de Cuentas Nacionales (SCN) y Cuentas Nacionales por Sectores Institucionales (CNSI) entre 1993 y 2002. Dicha tasa resultó de alrededor de 3 (o $300 \%$ ).

La tasa de plusvalor ajustada contrasta dramáticamente con la estimada en los orígenes de la presente investigación (Martínez, 1999), la cual resultó de entre 4 y 7, calculada con base en el sistema de SCN entre 1970 y 1990. Con tal sistema de SCN previo a 1993 no era posible excluir los ingresos de trabajadores autónomos de los excedentes de operación.

La modificación de los excedentes de operación explica sólo una parte de las diferencias entre la tasa de plusvalor ajustada y la no ajustada; otra parte se aclara por diferencias en la metodología con la cual se contabilizan las remuneraciones de asalariados en las cuentas del pasado y las actuales del SCN de México.

De manera que, al abordar empíricamente el problema del ingreso de los trabajadores autónomos en la estimación de la tasa de plusvalor de México, surgió un nuevo problema 


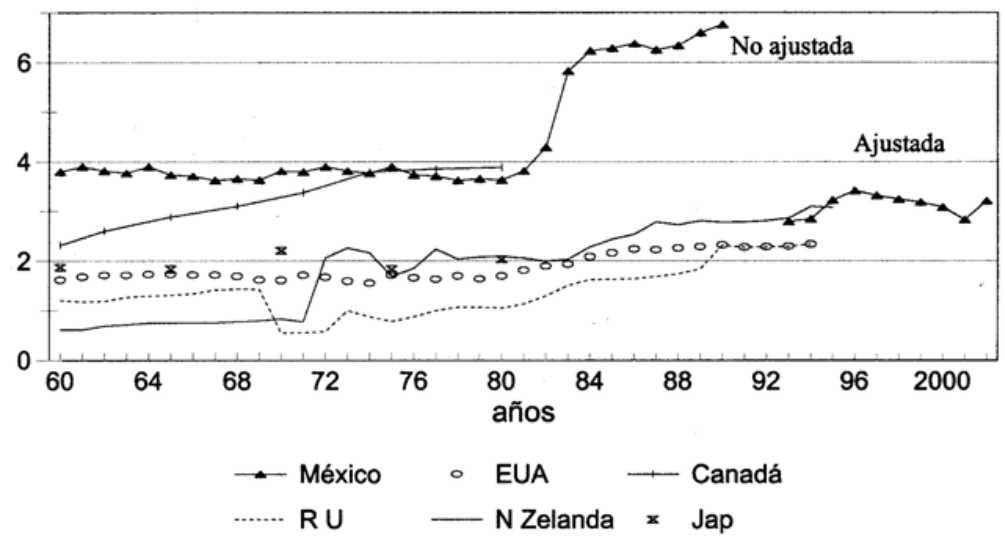

Gráfica 1. Tasas de plusvalor, México y países seleccionados.

Fuente: estimaciones propias para México basadas en estimaciones de Moseley (1991) para Estados Unidos (EU), de Murray (1993) para Canadá (CAN), de Cockshott (1995) para Reino Unido (RU), de Kalmans (1992) para Japón, y de Cronin (1998) para Nueva Zelanda.

empírico que se ha identificado pero no resuelto en esta etapa de la investigación: ¿por qué las magnitudes de remuneraciones de asalariados productivos son tan diferentes entre el SCN anterior a 1993 y el vigente a partir de dicho año?

El hallazgo original según el cual la tasa de plusvalor es mayor en México que en países desarrollados (Estados Unidos, Canadá, Reino Unido, Nueva Zelanda y Japón), parece que se modificaría sólo con respecto de Canadá, como se puede ver en la Gráfica 1. En virtud de que las tasas de plusvalor de aquéllos son de alrededor de 2 y la de éste llega a ser de 4, mientras que la nueva estimación de la de México es de alrededor de 3. Como está por verse si la metodología del SCN de México vigente desde 1993 para estimar la tasa de plusvalor es mejor que la previa, es posible que aún ajustando esta última, el hallazgo original no se modifique con respecto de Canadá ni en el periodo 1960-1990, cuando la tasa de plusvalor de México era más baja.

Veamos el problema con detalle. El ingreso de los trabajadores autónomos tiene una influencia en las estimaciones de la tasa de plusvalor por dos razones: porque representa una porción significativa del ingreso y del excedente de operación (total) y porque el ingreso de este tipo de trabajadores se registra como superávit de operación por el SCN de México. Por lo tanto, la cantidad correspondiente al plusvalor se sobreestima. No obstante, es posible que la conclusión principal, según la cual los trabajadores de un país subdesarrollado como México son más explotados que los trabajadores de naciones desarrolladas, se mantenga después de excluir el ingreso de los trabajadores autónomos del monto total de excedente de operación. ${ }^{4}$

4 En los países desarrollados, la cuestión de los trabajadores autónomos parece no constituir un problema importante en la estimación de la tasa de plusvalor, dado que este tipo de ingreso tiene un peso muy

\section{Desaarrollo}


Desde hace pocos años el Instituto Nacional de Estadística, Geografía e Informática de México produce las Cuentas Nacionales por Sectores Institucionales (CNSI), las cuales ya permiten separar el ingreso de los trabajadores autónomos de otros dentro del excedente de operación. ${ }^{5}$ Lamentablemente, no hay información desagregada por grandes divisiones de la actividad económica compatibles con el scN. Tal desagregación es importante para distinguir entre trabajo productivo e improductivo. Sin dicha desagregación, lo único que se puede hacer es un ajuste de los datos de SCN con base en las CNSI, en lo que se refiere al ingreso de los trabajadores autónomos.

Las primeras estimaciones de tasa de plusvalor monetaria de México (Martínez, 1999) usadas en esta investigación alcanzaban niveles de 7 (o 700\%) en 1990. Se trataba de estimaciones que excluían del análisis la gran división 1 (agropecuaria, silvicultura y pesca) con base en la consideración de que en dicha división el peso de los trabajadores autónomos era mucho mayor que en otras ramas y eso daría lugar a una sobreestimación aun mayor de la tasa de plusvalor, la cual iba a ser comparada con la correspondiente a países desarrollados. Los cálculos contabilizaban en el plusvalor: excedentes de operación (incluidos los ingresos de trabajadores autónomos), impuestos indirectos netos de subsidios y remuneraciones de asalariados de las grandes divisiones de la actividad económica consideradas improductivas. ${ }^{6}$ Mientras que en el capital variable se contabilizaban las remuneraciones de asalariados de las grandes divisiones restantes, catalogadas de productivas:

pequeño en los excedentes de operación. En un país subdesarrollado como México, corregir la sobreestimación de la tasa de plusvalor cambia el nivel de ésta pero no el hallazgo original. Con base en datos de la Encuesta Nacional de Ingreso y Gasto de los Hogares (ENIGH, 1989, cap. IV, p. 102), se estimó que la participación del ingreso de los trabajadores autónomos en el ingreso nacional era de $20.5 \%$ en 1989. Utilizando este cálculo se pudo excluir el ingreso de los trabajadores autónomos del excedente de operación del SCN mexicanas. Así, se encontró que la tasa de plusvalor disminuyó después de la ponderación (560\% antes y $441 \%$ después). El resultado alentador para esta investigación fue que la tasa de plusvalor en Estados Unidos permaneció más baja que en México. Sin embargo, las ENIGH se han publicado en forma discontinua en los últimos años y sus conceptos no han sido siempre los mismos; tienen problemas de subregistro de los ingresos de los hogares debido tanto a subdeclaraciones en las entrevistas y a truncamiento de datos de los estratos más pobres y más ricos causados por procedimientos de muestreo y, lo que es peor, no hay consenso sobre el mejor método para ajustar los datos a cuentas nacionales. Por lo anterior, es más recomendable trabajar con las CNSI que forman parte del SCN.

5 Por ejemplo, introducen el concepto de ingreso mixto, el cual representa el excedente derivado de las actividades productivas de una empresa no constituida en sociedad perteneciente a un hogar; el apéndice estadístico incluye las participaciones de los componentes de las cuentas de producción en el total de la economía y dentro de cada sector, en el cual vuelve a destacar el nuevo concepto de ingreso mixto al lado de remuneraciones y excedentes de operación. El ingreso mixto junto con el excedente de operación neto son los saldos contables de la cuenta de generación del ingreso; dichos saldos se definen como valor agregado menos remuneración a asalariados menos impuestos netos de subsidios sobre la producción.

6 Comercio, servicios financieros, seguros y bienes inmuebles, servicios profesionales, servicios de educación, servicios médicos, administración pública y defensa.

\section{Dêsaarróllo}


PLUSVALOR, INGRESO DE TRABAJADORES AUTÓNOMOS

\begin{tabular}{|c|c|c|c|c|c|}
\hline \multicolumn{6}{|c|}{$\begin{array}{c}\text { Cuadro } 1 \\
\text { Participación de los ingresos mixtos en valor agregado y excedentes de operación }\end{array}$} \\
\hline & $\begin{array}{c}\text { Excedente } \\
\text { neto de } \\
\text { operación }\end{array}$ & $\begin{array}{l}\text { Ingresos } \\
\text { mixtos }\end{array}$ & $\begin{array}{c}\text { Valor } \\
\text { agregado } \\
\text { neto total } \\
\text { economía total }\end{array}$ & $\begin{array}{c}\text { Cociente } \\
\text { ingresos mixtos/ } \\
\text { excedente de } \\
\text { operac. total }\end{array}$ & $\begin{array}{c}\text { Cociente } \\
\text { ingresos mixtos/ } \\
\text { valor agregado } \\
\text { neto }\end{array}$ \\
\hline 1993 & 597279384 & 246184949 & 1041744462 & 0.41 & 0.24 \\
\hline 1994 & 667538444 & 274363144 & 1176738566 & 0.41 & 0.23 \\
\hline 1995 & 889353560 & 359179922 & 1467992788 & 0.40 & 0.24 \\
\hline 1996 & 1284993114 & 508216458 & 2023592206 & 0.40 & 0.25 \\
\hline 1997 & 1596349314 & 627684507 & 2549765766 & 0.39 & 0.25 \\
\hline 1998 & 1928081066 & 749723696 & 3120557614 & 0.39 & 0.24 \\
\hline 1999 & 2289081851 & 894893394 & 3743844081 & 0.39 & 0.24 \\
\hline 2000 & 2711934484 & 111822004 & 74456970692 & 0.41 & 0.25 \\
\hline 2001 & 2781044126 & 1177544310 & 4702788721 & 0.42 & 0.25 \\
\hline
\end{tabular}

Montos expresados en miles de nuevos pesos corrientes.

Fuente: elaboración propia con base en INEGI, Sistema de Cuentas Nacionales y por Sectores Institucionales de México, 2004.

minería, manufactura, construcción, electricidad, restaurantes y hoteles, transporte, almacenamiento y comunicaciones.

Las estimaciones basadas en el SCN y de CNSI a partir de 1993 difieren de las anteriores, en las cuales incluyen la gran división 1 de la actividad económica y se excluyen los ingresos de los trabajadores autónomos (denominados ingresos mixtos) de los excedentes de operación. Como se puede ver en el Cuadro 1, los ingresos mixtos representan al rededor del 0.40 de los excedentes de operación y del 0.24 del valor agregado neto, por lo que la exclusión de dichos ingresos afecta notablemente el nivel de la tasa de plusvalor.

En el Cuadro 2 (A, B y C) se resumen las diversas estimaciones de dichas tasas en un año de traslape (1993) de los SCN.

Las estimaciones del Cuadro 2B son congruentes con las estimaciones de Martínez (1999); se basan en el Sistema de Cuentas Nacionales previo a 1993. Los excedentes de operación no están ajustados y si se excluye la gran división 1, la tasa de plusvalor es de 7 en 1990 y 1993, respectivamente.

Las estimaciones del cuadro 2A, que sí ajustan la tasa de plusvalor mediante la exclusión de los ingresos de autónomos de los excedentes de operación, difieren de las de Martínez (1999) en 400\%, según se puede comparar con el cuadro 2B. La exclusión de la gran división 1 en las estimaciones de Martínez (1999) explica sólo 100\%.7 ¿Qué explica la diferencia de $300 \%$ cuando ya se incluye a la gran división 1 ?

7 Se ha dicho que las estimaciones de tasa de plusvalor de México de Martínez (1999) excluyen la gran división 1 debido al gran peso del trabajo autónomo en esa división, de manera que para poder comparar los cálculos posteriores se tiene que examinar si la inclusión o exclusión de dicha división provoca cambios importantes en la tasa de plusvalor.

\section{Desarrrollo}


Cuadro 2

Tasa de plusvalor. Comparación de componentes, 1993. Según distintas fuentes, se ajustan excedentes de operación excluyendo ingresos de cuentapropistas o excluye gran división 1

\begin{tabular}{|c|c|c|}
\hline \multicolumn{3}{|c|}{ Parte A } \\
\hline \multicolumn{3}{|c|}{$\begin{array}{l}\text { Cuentas Nacionales y Cuentas Nacionales } \\
\text { por Sectores Institucionales, 1993-2002, } \\
\text { excedentes de operación ajustados, } \\
\text { incluye Gran División 1. Excluye GD1 }\end{array}$} \\
\hline Capital variable & 210317825 & 197568009 \\
\hline Plusvalor & 585241688 & 597991504 \\
\hline Tasa de plusvalor & 2.78 & 3.03 \\
\hline \multicolumn{3}{|c|}{ Parte B } \\
\hline & $\begin{array}{c}\text { Cuentas Nacionales } \\
1988-1993\end{array}$ & $\begin{array}{c}\text { Cuentas Nacionales } \\
1988-1993\end{array}$ \\
\hline & Incluye $G D 1$ & Excluye $G D 1$ \\
\hline Capital variable & 143269707.00 & 131884646.00 \\
\hline Plusvalor & $\begin{array}{lll}871 & 433 & 127.00\end{array}$ & $\begin{array}{llll}882 & 818 & 188.00\end{array}$ \\
\hline Tasa de plusvalor & 6.08 & 6.69 \\
\hline \multicolumn{3}{|c|}{ Parte $C$} \\
\hline & $\begin{array}{c}\text { Cuentas Nacionales } \\
1988-2002\end{array}$ & $\begin{array}{c}\text { Cuentas Nacionales } \\
1988-2002\end{array}$ \\
\hline & Incluye $G D 1$ & Excluye $G D 1$ \\
\hline Capital variable & 210317825.00 & 197568009.00 \\
\hline Plusvalor & 831426637.35 & 844176453.35 \\
\hline Tasa de plusvalor & 3.95 & 4.27 \\
\hline
\end{tabular}

Exceptuando las tasas, los montos están expresados en miles de pesos corrientes.

Fuente: elaboración propia con base en INEGI, Sistema de Cuentas Nacionales de México, 1995 y 2004 .

Para responder esa pregunta, veamos: las estimaciones de los cuadros $2 \mathrm{~B}$ y $2 \mathrm{C}$, que auqnue debieran ser iguales, en ningún caso se ajustan los excedentes de operación, sin embargo, difieren: si se incluye la gran división 1 difieren en $200 \%$ y si se excluye, difieren en 300\%. Las primeras estimaciones se basan en datos del SCN previo a 1993, mientras que las segundas en el SCN vigente a partir de 1993. Es decir, por el hecho de basarse en fuentes diferentes, las estimaciones cambian entre 200 y $300 \%$.

Se puede observar en los cuadros $2 \mathrm{~B}$ y $2 \mathrm{C}$ que las diferencias significativas se encuentran entre los montos de capital variable, esto es, entre los montos de remuneraciones de asalariados de las grandes divisiones productivas de la actividad económica. Las del siste- 
ma vigente desde 1993 representan $150 \%$ de las correspondientes al sistema previo, mientras que los excedentes de operación del sistema vigente representan 0.95 de los del sistema previo. Así que se tiene un nuevo problema empírico: ¿por qué las magnitudes de remuneraciones de asalariados productivos son tan diferentes entre el SCN anterior a 1993 y el vigente? En un trabajo posterior se debiera examinar qué cambios en la metodología de la contabilidad de las remuneraciones de asalariados aclaran la diferencia.

Se puede afirmar que la modificación de los excedentes de operación explica sólo una parte de las diferencias entre la tasa de plusvalor ajustada y la no ajustada. Otra parte de las distinciones se explica en la metodología con la cual se contabilizan las remuneraciones de asalariados en las cuentas del pasado y las cuentas actuales del SCN de México.

De acuerdo con la nueva estimación de la tasa de plusvalor de alrededor de 3 , ajustada y que se basa en los datos del SCN vigente, el hallazgo original según el cual la tasa de plusvalor es mayor en México que en países desarrollados (Estados Unidos, Canadá, Reino Unido, Nueva Zelanda y Japón) parece modificarse sólo con respecto de Canadá. Esto, si las tasas de plusvalor de dichos países permanecen cerca de 2 y la de Canadá alrededor de 4 al inicio de los noventa.

En tanto no se aclaren las diferencias entre las metodologías de los distintos SCN de México no se puede admitir que las estimaciones de tasa de plusvalor de Martínez (1999) deban reducirse más allá de lo atribuible a la sobreestimación del plusvalor debido a la contabilidad del ingreso de autónomos en los excedentes de operación. En estas condiciones, el hallazgo original tampoco se modificaría con respecto de Canadá, ni aun en el periodo 1960-1990, cuando la tasa de plusvalor de México era más baja.

\section{Aspectos teóricos acerca de la tasa de plusvalor y trabajo autónomo}

Anteriormente se vio cómo diversos autores han tratado de manera empírica los ingresos de los trabajadores autónomos. Enseguida se verá cuáles han sido sus razones teóricas o cuáles se pudieron inferir cuando no las hicieron explícitas y las implicaciones de esto.

Partiendo de la revisión que hace Cámara (2003) de distintos tratamientos que se han hecho de los ingresos de los trabajadores autónomos en algunos desarrollos sobre tasa de plusvalor, se distingue que varios casos consisten en procedimientos de cuantificación del capital variable o de la tasa de plusvalor, que no tratan específica y teóricamente el problema. En dichos casos se pueden señalar algunas implicaciones teóricas a partir de sus ejercicios contables. Según se vio anteriormente, los tratamientos se pueden agrupar en dos enfoques: el que se referirá como dominante por predominar en la literatura empírica, planteado por Shaikh y Tonak, de acuerdo con el cual el valor nuevo que produce un trabajador autónomo se puede dividir entre capital variable y plusvalor; y el alternativo — propuesto por Guerrero, seguido por Cámara y que se comparte en esta investigación-, de acuerdo

\section{Desarrerrollo}


con el cual dicho valor nuevo es un componente de la renta que posee identidad propia y se debe separar del valor nuevo producido en forma capitalista.

Según Cámara (op. cit.), el carácter capitalista no se define con base en el mero ánimo de lucro como en el apartado anterior parecería seguirse equivocadamente de la afirmación de Shaikh y Tonak (1994), sino con base en la utilización de trabajo asalariado. En este artículo no se discute la conveniencia de llamar capitalista a un tipo de producción y no a otro, sólo se distingue lógicamente entre el excedente producido con base en trabajo asalariado y el generado por la existencia de este último.

\section{Enfoque dominante}

Shaikh y Tonak y los autores que siguen su metodología comparten este enfoque. ${ }^{8}$ De acuerdo con ellos, la producción mercantil del trabajo no asalariado es un tipo especial de producción capitalista. Siguiendo a Cámara, proceden como si el trabajador autónomo, dueño de medios de producción, se contratara y explotara a sí mismo, por lo cual el valor nuevo que creará se pudiera dividir entre capital variable y plusvalor. Recuérdese que para estos autores:

Ignorar el equivalente salarial de las personas autoempleadas (propietarios y socios) equivale a tratar todo el valor añadido como renta de tipo beneficio. Hemos optado en cambio por imputar un equivalente salarial al trabajo de las personas autoempleadas y tratar el resto del valor añadido como renta de tipo beneficio (que puede ser negativa en caso de pérdidas) (Shaikh y Tonak, 1994:112n).

Por ejemplo, para Rebeca Kalmans la tasa de explotación concierne a los trabajadores productivos empleados por empresas capitalistas y trabajadores autoempleados. No se encontraron sus razones teóricas, sólo su consideración práctica: el que este tipo de ingreso aparezca en los excedentes de operación obliga a imputar un salario equivalente y sumarlo al capital variable para evitar inflar la tasa de plusvalor.

Aunque Gouverneur (2002) aporta una reflexión teórica extensa acerca del problema, su postura, vinculada a su ejercicio empírico, resulta muy próxima al enfoque dominante, pues acuña las categorías plusvalor y capital variable. En éstos incluye el rédito cedido por los trabajadores autónomos a los capitalistas en la circulación y el ingreso que los primeros perciben, respectivamente. Gouverneur (1990) utiliza el mismo método que Sahikh y Tonak: divide la renta de la producción mercantil no capitalista entre capital variable y plusvalor, pues imputa un equivalente salarial a los trabajadores independientes y lo suma al capital variable para calcular la tasa de plusvalor.

Dice que todo el trabajo que se realiza en el sector mercantil produce valor.

8 Se ha proporcionado un panorama del tratamiento empírico del problema en el apartado anterior.

\section{Desarrrollo}


todos los trabajadores en el sector mercantil toman parte en la producción de mercancías y de valor, y los procesos de producción realizados en las empresas deben entenderse en un sentido mucho más amplio que el de la mera transformación de insumos en producto (Gouverneur, 2002:28-29).

Él caracteriza al trabajo autónomo — que llama independiente- de la siguiente manera:

Los independientes también tienen dos características distintivas: 1. Al igual que los capitalistas, poseen medios de producción (empresas) y viven, no de la venta de su fuerza de trabajo, sino de la venta de bienes o servicios producidos en su empresa. 2. A diferencia de los capitalistas, no compran la fuerza de trabajo de asalariados para producir dichos bienes o servicios, sino que los producen personalmente. (Esto no excluye que los independientes puedan ser ayudados por familiares los "ayudantes" pero la fuerza de trabajo de éstos no es comprada en el mercado laboral.)

Todos los independientes se encuentran vinculados al sector de producción mercantil (trabajo indirectamente social). Con el fin de mostrar tanto la analogía como la diferencia con los capitalistas, pueden llamarse también productores mercantiles simples: "mercantiles" debido a que producen para la venta; "simples" porque no dependen de la compra de fuerza de trabajo (Gouverneur, op. cit.:2002:16).

Gouverneur (ibid.) plantea que los trabajadores independientes tienen mayor capacidad para reproducirse que las empresas capitalistas, pues ellos no precisan la ganancia media para desempeñar su actividad. Eso es inexacto: sólo si fueran más productivos que el promedio de la rama en la cual producen estarían en mejores condiciones que las empresas capitalistas de similar productividad para afrontar tiempos difíciles. Pero en condiciones normales o de auge, difícilmente estarán mejor que estas empresas de similar productividad pues no acumulan igual. Claro está que todo esto es hipotético pues los trabajadores autónomos generalmente tendrán menor productividad que el promedio y, en tal caso, su permanencia como productores dependerá de qué tanto puedan comprimir sus necesidades para subsistir.

Gouverneur afirma que los trabajadores autónomos transfieren rédito a las empresas capitalistas y que la ganancia capitalista es mayor que la plusvalía producida por los asalariados que trabajan en estas empresas. ${ }^{9}$

A esta fuente primaria de la ganancia ${ }^{10}$ debe agregarse otra: la parte del rédito perdida por los productores independientes que se enfrentan con empresas capitalistas que utilizan técnicas más avanzadas o tienen un mayor poder de mercado. Esta segunda fuente de ganancia no es típica del capitalismo: en este caso, la ganancia no surge de las relaciones de

9 Si eliminamos el supuesto de un sistema exclusivamente capitalista y consideramos la existencia de sectores de producción no capitalista, aparecen diferencias entre plusvalía y ganancia, incluso en el nivel macroeconómico: a) debido a las transferencias de rédito de los productores independientes, la ganancia es mayor que la plusvalía creada por los asalariados (cap. VI, 1.3.2.a); b) debido a las exacciones públicas destinadas a financiar las instituciones de interés público, la ganancia disponible es menor que la plusvalía creada (cap. VI, 3.3.1), Gouverneur (2002:54, nota 6).

10 Gouverneur se refiere a la plusvalía producida por los asalariados.

\section{Desarrrollo}


explotación en la producción, sino de las relaciones de competencia y poder en el mercado. Si tomamos en cuenta la presencia de productores independientes, la ganancia total $(\mathrm{G})$ es, entonces, igual al total de plusvalía creada $(\mathrm{Pv})$, más el rédito creado — pero perdido— por los productores independientes ("Pv"). Tenemos entonces:

$$
\mathrm{G}=\mathrm{Pv}+\text { "Pv" }
$$

Como ya se dijo (capítulo V, sección 1), la plusvalía total $(\mathrm{Pv})$ depende del número total de asalariados que toman parte en la producción de mercancías, de la duración promedio de su jornada laboral y del salario promedio (la duración del trabajo presente determina el rédito creado por cada uno, el nivel del salario determina la distribución de este rédito entre la remuneración del trabajador y la plusvalía). De manera análoga, la "plusvalía" ("Pv") depende del número total de productores independientes, de la duración promedio de su jornada laboral y del rédito promedio (Gouverneur, ibid::95-96).

En esta investigación se entiende que el trabajo de los autónomos pasa por el mercado, es decir, es trabajo cuya regulación se realiza mediante el mercado y no en la esfera individual o familiar. Se contabiliza como trabajo desempeñado en la esfera capitalista y recibe así la sanción social. Si los trabajadores autónomos son más eficaces que el promedio, son premiados, y lo contrario ocurre cuando son menos productivos. Pero me parece que esto no permite concluir que los trabajadores autónomos, menos productivos que los asalariados, ceden rédito a los capitalistas.

\section{Enfoque alternativo}

De acuerdo con dicho enfoque, el ingreso del trabajo autónomo no es capital variable ni plusvalor. Smith (1993) establece que para Marx hay dos principales formas de labor improductiva, dentro de las cuales está el conjunto de actividades socioeconómicas no capitalistas y que, por lo tanto, no sirven para incrementar el plusvalor social (el trabajo de productores autoempleados es la principal forma). Guerrero (1989) y Cámara (2003) así lo consideran y agregan que dicho ingreso posee una identidad propia.

Según Guerrero (1989), para estudiar fenómenos como la distribución de la renta y su relación con la producción y la acumulación capitalistas, se requiere delimitar el sector capitalista con respecto del sector formado por sujetos de un modo de producción mercantil simple precapitalista, coexistente pero lógica e históricamente precedente. Después de excluir la parte de la renta nacional que corresponde a empresarios sin asalariados y trabajadores independientes, es posible referir exclusivamente la renta nacional aportada por el modo de producción capitalista y aplicar la noción de Marx de que todo el valor nuevo creado anualmente (valor añadido) que excede del valor de la fuerza de trabajo es el plusvalor.

Guerrero (2005) establece que la explotación tiene que ver con la participación de los asalariados en la renta nacional; que todo aquel que sostiene una relación salarial con un

\section{Desarrrollo}


patrón (sea éste privado o el Estado) es, por definición, proletario; que los trabajadores autónomos (trabajadores independientes y empresarios sin asalariados) no son capitalistas ni asalariados. El caso más claro de trabajo que se lleva a cabo en la esfera mercantil pero no es productivo de plusvalor o, dicho de otra forma, que produce valor más no plusvalor, es el de los trabajadores autónomos.

Guerrero afirma que la capa intermedia de la población formada por los trabajadores autónomos situados entre las clases capitalista y obrera se ha identificado tradicionalmente con el llamado modo de producción mercantil simple. Un modo de producción relacionado con el capitalista, pero diferente de él. Según el autor, se trata de una oposición conceptual que no excluye la simultaneidad histórica de los dos modos de producción.

En primer lugar, la clase obrera o proletariado puede identificarse con la suma de asalariados ocupados (tanto públicos como privados) y de parados [...] la burguesía se identifica con los empleadores, porque nos parece que el criterio decisivo para distinguir a esta clase de la de la pequeña burguesía es el de si ejercen, o no, su dominio sobre al menos un asalariado; pues es este dominio, precisamente, lo que convierte los medios de producción en capital. Como es lógico, puede haber pequeños empleadores con un nivel de renta inferior al de determinados empresarios sin asalariados (sobre todo, en las ramas de más alto nivel de productividad); pero ello no significa nada en relación con la cuestión de la definición de clases, como tampoco lo significa el hecho de que algunos asalariados perciban igualmente una renta superior a la de algunos pequeños empleadores (con o sin asalariados). Por último, en la pequeña burguesía incluimos al resto de la población activa, es decir, a los "ESATI" (empleados sin asalariados y trabajadores independientes) y a los "ayudas familiares" ; pues suponemos que estos últimos lo son, fundamentalmente, de los primeros, aunque una determinada proporción de los mismos estén vinculados también a pequeños empleadores (1989:238).

\section{Una nota para contribuir al examen teórico de plusvalor y trabajo autónomo}

Con Gouverneur (2002) se veían ya las posibles complejidades del problema del cálculo de la tasa de plusvalor, una vez que se considera el trabajo autónomo. Con las reflexiones de este apartado se pretende mostrar las dificultades que introduce el trabajo autónomo en el cálculo de la tasa de plusvalor.

Dentro del conjunto de trabajadores autónomos hay que distinguir dos tipos: 1) los asalariados disfrazados de autónomos, trabajadores subcontratados por capitalistas, que por esa circunstancia son, en realidad, asalariados, y 2) los autónomos puros, es decir, trabajadores auténticamente no asalariados. El plusvalor generado por los primeros aparece en el valor agregado de las empresas capitalistas que los subcontratan; el ingreso que perciben debe sumarse a las remuneraciones de trabajadores productivos para estimar el capital variable. Dentro del segundo grupo, hay cuatro subgrupos:

a) Los que compiten con empresas capitalistas y tienen una productividad menor a la promedio de dichas empresas. Al parecer, éstos tienen una tasa de ingreso menor que la expresión

\section{Desaarrollo}


dineraria del valor y producen un excedente que hay que imputar con base en esa diferencia y descontar del valor agregado, para estimar la tasa de plusvalor. $b$ ) Los que compiten con empresas capitalistas con una productividad igual a la promedio de tales empresas. En este caso, bastaría con descontar sus ingresos del valor agregado para estimar la tasa de plusvalor. c) Los trabajadores autónomos que no compiten con empresas capitalistas. Como en el caso anterior, se deben descontar sus ingresos del valor agregado para estimar la tasa de plusvalor. d) Los trabajadores autónomos cuya tasa de ingreso es superior a la expresión dineraria del valor, que se apropian de plusvalor. Habría que excluir sus ingresos con base en el número total de trabajadores por la expresión dineraria del valor, pues el resto sería plusvalor apropiado por una parte de esos trabajadores. Se considera que se trata de trabajadores calificados que se apropian de plusvalor en una magnitud que no se explica por el hecho de emplear asalariados; ni el valor que se apropian se justifica por su calificación.

Así que, cuando se trata de verdaderos trabajadores autónomos, la masa de ingresos de éstos no es siempre equivalente al valor nuevo de la producción mercantil no capitalista (VNpmnc). Para que lo sea, se requiere que tales autónomos no compitan con empresas capitalistas o que, haciéndolo, lo realicen con la productividad promedio de sus competidoras.

En suma, el excedente capitalista producido en unos casos o apropiado en otros por los trabajadores auténticamente no asalariados habría que calcularlo con base en la diferencia entre su tasa de ingreso y la expresión dineraria del valor de la economía. Es decir, para estimar la tasa de plusvalor en una economía real, no sería suficiente con excluir del excedente de operación una masa de ingresos con base en el número de ESATIS y una tasa salarial promedio.

En una economía en la cual los trabajadores autónomos no intercambian el producto de su trabajo, resulta evidente que este tipo de trabajadores no son explotados. Que no lo sean también es evidente en una economía en la cual el trabajo autónomo produce, en promedio, la misma productividad que el trabajo asalariado.

En una economía en la cual coexisten trabajadores autónomos menos productivos que los trabajadores asalariados, ya se puede plantear el problema de si los primeros son explotados. Veamos una economía hipotética en la cual el subsector 1.1 está conformado por trabajadores autónomos; el subsector 1.2 y el sector 2 usan trabajo asalariado para producir maíz y fertilizante respectivamente, y la productividad del subsector 1.1 es menor que la del subsector 1.2. Suponiendo unas técnicas —que aquí no es necesario explicarconsistentes con las cracterísticas mencionadas, el Cuadro 3 muestra la matriz de transacciones intersectoriales en valor de dicha economía.

Se tiene el valor de la mercancía $1, \mathrm{~m} 1=1.12 \mathrm{aH} / \mathrm{tn}$ maíz; en virtud de que el recíproco del valor de una mercancía, la productividad, nos dice cuál es el consumo máximo posible por trabajador, éste es 1/1.12 aH/tn maíz = 0.89 tn maíz/año hombre de trabajo $(\mathrm{aH})$; hay 
PLUSVALOR, INGRESO DE TRABAJADORES AUTÓNOMOS

Cuadro 3

Matriz de transacciones intersectoriales en valor de una economía hipotética

\begin{tabular}{lrrr}
\hline & 1 & 2 & Total \\
\hline 1 & 84 & 0 & \\
2 & 60 & 0 & \\
Años hombre & 80 & 20 & \\
Salarios & 24 & 12 & 36 \\
Ingresos au & 16 & 0 & 16 \\
Excedente & 40 & 8 & 48 \\
Valor agregado & 80 & 20 & 100 \\
Producción bruta & 20 & 224 & 244 \\
\hline
\end{tabular}

aH: años hombre de trabajo

aH se dividen: 40 en el subsector 1.1; 40 en el subsector 1.2 y 20 en el sector 2 . au: trabajadores autónomos

una tasa salarial del 0.6 del consumo máximo posible de 0.89 tn $\mathrm{m} / \mathrm{aH}: 0.5357 \mathrm{tn} \mathrm{m} /$ aH(asalariado) y una tasa de ingreso autónomo de 0.3571 tn m/aH (autónomo).

En una economía en la cual rigen precios se puede considerar que éstos son proporcionales a los valores de las mercancías. Es decir, $\mathrm{p}_{\mathrm{i}}=\alpha \mathrm{m}_{\mathrm{i}}$, donde $\alpha$ es la expresión dineraria del valor. Se puede probar que si los precios son proporcionales a los valores, el valor agregado por hombre ocupado mide la productividad y es igual a la expresión dineraria del valor. Cuando la tasa salarial es inferior a ésta, el ingreso de los trabajadores no puede comprar todo el producto social, hay otro ingreso distinto al salario, el excedente capitalista; se revela la explotación del trabajo asalariado. De ahí la insistencia en que el excedente capitalista producido en unos casos o apropiado en otros, por los trabajadores auténticamente no asalariados, se estime con base en la diferencia entre su tasa de ingreso y la expresión dineraria del valor de la economía.

Volviendo al ejemplo, se puede calcular la variedad de tasas de explotación que se enumeran a continuación:

“Tasa de plusvalor”, Gouverneur

$$
\begin{array}{ll}
= & \text { plusvalor }+ \text { "plusvalor" / capital variable + "capital variable" } \\
= & \text { trabajo excedente / trabajo necesario para asalariados y autónomos } \\
= & 48 / 52 \quad=\quad 0.9231
\end{array}
$$

Tasa de plusvalor, Shaikh-Tonnak =

trabajo excedente ajustado/trabajo necesario para asalariados ajustado

$$
=18.7 / 60=0.3111
$$

para la cual se hacen los siguientes ajustes:

\section{Desaarrollo}


Ajuste de trabajo excedente:

Excente en valor + (ingresos autónomos - salarios imputados a autónomos)

Excedente en valor + (ingresos autónomos - $($ tasa salarial $\left.\left.)(\mathrm{AH}(\alpha v)) \mathrm{m}_{1}\right)=18.6643\right)$

Ajuste de trabajo necesario para asalariados:

Salarios en valor + salarios imputados a autónomos $\left(\mathrm{m}_{1}\right)$

Salarios en valor $+($ tasa salarial $\left.* \mathrm{AH}(\alpha v)) \mathrm{m}_{1}=60\right)$

Tasa de plusvalor, Guerrero-Cámara

trabajo excedente total/trabajo necesario para asalariados o

trabajo excedente atribuible a autónomos y a asalariados/ trabajo necesario para asalariados

$$
=48 / 36=1.33
$$

Hasta donde se ha podido ver, Guerrero y Cámara restan el ingreso de los trabajadores autónomos del excedente de operación de las cuentas nacionales reales, pero no restan el excedente atribuible a los autónomos. Por tal razón, en este ejemplo calcularían así la tasa de plusvalor.

En este ejemplo numérico, si se quiere excluir el trabajo autónomo de la estimación de la tasa de plusvalor, es necesario restar el trabajo excedente atribuible a éste, del trabajo excedente. Así se tiene el excedente en $t n$ de $m$ atribuible al trabajo autónomo:

Por trabajador: consumo máximo posible - tasa de ingreso autónomo $=0.5357$ y el total: (consumo máximo posible - tasa de ingreso autónomo) $(\mathrm{aH}(\mathrm{au}))=$

21.4286

Por su parte, el trabajo excedente atribuible a (el trabajo o el intercambio de) los autónomos:

$$
(\text { Total })\left(\mathrm{m}_{1}\right)=24 \mathrm{aH}
$$

La propuesta propia de tasa de plusvalor, la tasa de explotación exclusivamente de trabajadores asalariados ya se puede calcular como:

trabajo excedente atribuible a trabajadores asalariados/ trabajo necesario para traba-

$$
\text { jadores asalariados }=24 / 36=0.6667 \mathrm{aH}
$$

Este ejemplo ilustra que en una economía en la cual coexisten trabajadores asalariados y trabajadores autónomos menos productivos que los primeros puede haber un excedente atribuible a los segundos. Pero esto no significa que los trabajadores autónomos son explotados. Se sabe que a la circulación no puede tener más valor que el que se produjo en la producción. En esta economía, un más de valor en el subsector de trabajo asalariado

\section{Desaarrollo}


corresponde con un menos de valor en el subsector de trabajo autónomo. El trabajo de los autónomos pasa por el mercado, es decir, es trabajo cuya regulación se realiza mediante el mercado y no en la esfera individual o familiar. Se contabiliza como trabajo desempeñado en la esfera capitalista y recibe así la sanción social. Si los trabajadores autónomos son más eficaces que el promedio, son premiados, y lo contrario ocurre cuando son menos productivos que el promedio. Aquí los trabajadores autónomos son castigados por su menor productividad, el trabajo social que les es reconocido en el mercado, o dicho de otra forma, la magnitud de trabajo social que les reconoce el mercado es menor que la magnitud de su trabajo individual.

No se puede afirmar que los trabajadores autónomos son explotados o explotados en un sentido amplio, como se sigue de lo expuesto por Gouverneur (2002); los cuales siendo menos productivos que los trabajadores asalariados generan más valor y, por lo tanto, transfieren valor a los capitalistas del sector donde los segundos producen. Este problema es el mismo que está presente en la discusión sobre si trabajadores menos productivos transfieren valor a otros más productivos dentro de una rama. A mi parecer, la solución de este problema no está terminada y ello impide resolver en su totalidad el que aquí nos ocupa.

\section{Conclusiones}

En este trabajo se ha planteado que si bien la existencia de trabajadores autónomos cambia el nivel de la tasa de plusvalía, se sigue manteniendo una conclusión previa: que el país atrasado, México, resulta más explotado que el país adelantado, Estados Unidos. La tasa de plusvalor ajustada al tomar en cuenta el trabajo autónomo contrasta dramáticamente con la estimada sin hacerlo (Martínez, 1999). No obstante, de acuerdo con la nueva estimación de la tasa de plusvalor de alrededor de 3, ajustada, el hallazgo original — la tasa de plusvalor es mayor en México que en países desarrollados (Estados Unidos, Canadá, Reino Unido, Nueva Zelanda y Japón) — cambia sólo con respecto de Canadá.

Para llegar a estos resultados analizamos la metodología de Shaikh y Tonak (1994). Sostuvimos que dicho enfoque es incorrecto, pues distribuye los ingresos de los trabajadores autónomos entre capital variable y plusvalor para estimar la tasa de plusvalor. Al hacerlo así, trata al trabajo autónomo como sujeto a explotación. No se encontraron las razones teóricas de estos autores, sino sólo consideraciones prácticas.

En este trabajo se comparte el enfoque alternativo de Guerrero (1989) y Cámara (2003), de acuerdo con el cual los ingresos de los trabajadores autónomos no son capital variable ni plusvalor, por lo cual, para estimar la tasa de plusvalor, es necesario excluir dichos ingresos del análisis.

Nuestro resultado parece contravenir la lógica impecable de Marx: la mayor productividad precisa de una composición orgánica más elevada y ello exige una mayor tasa de plusvalía. ¿Por qué entonces, empíricamente, resultan más altas las tasas de plusvalor en

\section{Desaarrollo}


México que en Estados Unidos? Esta pregunta puede plantearse mucho más firmemente después de la investigación que se describe en el artículo. No tenemos una respuesta a dicha pregunta, pero es importante destacar que la investigación científica avanza generalmente con base en preguntas formuladas claramente.

Si nuestros resultados son una norma del capitalismo contemporáneo y no una excepción, además de tratar de entender por qué éste opera así tendremos que examinar las explicaciones convencionales de que en México falta excedente y, por ello, deben atraerse inversión y ahorro foráneos.

Finalmente, nuestra investigación marcha en el camino trazado por muchos teóricos latinoamericanos: el atraso económico es un fenómeno estructural que nos obliga a estudiar nuestra realidad con un enfoque propio.

Bibliografía

Amin, Samir, ¿Cómo funciona el capitalismo? El intercambio desigual y la ley del valor, México, Siglo XXI, 1975.

Amsden, Alice, "An International Comparison of the Rate of Surplus Value in Manufacturing Industry", en Cambridge Journal of Economics, núm. 5, 1981, pp. 229-249.

Bettelheim, Charles, "Observaciones teóricas", en A. Emmanuel, El intercambio desigual, México, Siglo XXI, 1972, pp. 305-359.

Cámara Izquierdo, Sergio, "Tendencias de la rentabilidad y de la ley de acumulación de capital en España 1954-2001", tesis doctoral, Madrid, Facultad de Ciencias Políticas y Sociología, Departamento de Economía Aplicada V, Universidad Complutense de Madrid, 2003.

CEPAL, Panorama social de América Latina 20012002, Santiago de Chile, 2003.

Cronin, Bruce, "Productive and Unproductive Capital: a Mapping of the New Zeland System of National Accounts to Classical Economic Categories, 1972-1995", en Review of Political Economy, vol. 13, num. 3, 2001, pp. 309-327.

, "The Rate of Surplus Value in New Zeland", 1999, inédito.

Cockshott, Paul; Allin Cottrell y Greg Michaelson, "Testing Marx: Some New Results from UK Data”, en Capital and Class, vol 55, primavera de 1995, pp. 103-129

, "A Replay to Maniatis", en Capital and Class, vol. 59, verano de 1996, pp. 55-59.

Emmanuel, A., El intercambio desigual, México, Siglo XXI, 1972.
Gouverneur, Jacques, Comprender la economía, Lovaina, Diffusion Universitaire CIACO, edición electrónica, 1998.

"Productive Labor, Price/Value Ratio and Rate of Surplus Value: Theoretical View Points and Empirical Evidence", en Cambridge Journal of Economics, núm. 141, 1990, pp. 1-27.

Guerrero, Diego, La explotación. Trabajo y capital en España (1954-2001), 2005, en prensa.

, "Acumulación de capital, distribución de la renta y crisis de rentabilidad en España (19541987)", tesis doctoral, Madrid, Universidad Complutense de Madrid, Colección Tesis Doctorales, núm. 86/89, 1989.

Guerrero, Diego y Emilio Díaz C., "The Welfare State in the Distribution of National Income in Spain since the Transition", en International Journal of Political Economy, vol. 27, núm. 4, invierno de 1997-98, pp. 80-95.

Izumi, H., "International Comparisons of the Rate of Surplus Value using Labor Values", en Keizai, núm. $227, \mathrm{~s} / \mathrm{f}$.

Kalmans, R., "The Political Economy of Exploitation: A Comparative Study of The Rate of Surplus Value in Japan and The United States, 1950 1980", disertación doctoral, Nueva York, Department of Economics, New School for Social Research, 1992.

Lenin, V.I., Sobre el problema de los mercados, t. 3 , México, Siglo XXI, 1974.

Luxemburgo, Rosa, La acumulación del capital, 3a. parte, caps. XXV-XXXII, México, Grijalbo, 1967, pp. 251-352. 
_, y Nicolai Bujarin, "El imperialismo y la acumulación del capital. Buenos Aires, Ediciones del Pasado y Presente, 1975.

Mage, Shane, "The 'Law of the Falling Rate of Profit': its Place in the Marxian Theoretical System and Relevance to the United States", disertación, Nueva York, Columbia University, 1963.

Mandel, Ernest, Tratado de economía marxista, t. II, cap. x, México, Era, 1972, pp. 281-318.

Marini Ruy, Mauro, Dialéctica de la dependencia, cap. I, México, Era, 1989.

Martínez González, B. Gloria, "Algumas evidências da superexploracão nos países subdesenvolvidos: a atualidade do pensamento de Marini”, en Revista da Sociedade Brasileira de Economia Politica, num. 4, junio, 1999, pp. 105-121.

, "The Industrial Relative Wage in Mexico, 1960-1990", en International Journal of Political Economy, vol. 27, núm. 4, invierno de 19971998, pp. 80-95.

, "Una comparación del salario relativo entre México y Estados Unidos, 1960-1990", en Economía: Teoría y Práctica, nueva época, núm. 6, UAM-X, 1996, pp. 77-86.

Marx, Karl y Federico Engels, Teorías sobre la plusvalía, t. I, México, FCE, 1987, pp. 61-271.

Mateo Tomé, Juan Pablo, "Análisis de la evolución y el papel de la relación salarial en la economía de Venezuela durante el período de crisis y reestructuración (1979-1996)", investigación presentada para la obtención del diploma de Estudios Desarrollados, Madrid, Dpto. de Economía Aplicada, Doctorado en Economía Internacional y Desarrollo, Facultad de Ciencias Económicas y Empresariales, Universidad Complutense de Madrid, 2003.
Moseley, Fred, "Unproductive Labor and the Rate of Profit: a Replay", en Science and Society, vol. 58, núm. 1, 1994, pp. 84-92.

, The Falling Rate of Profit in the Postwar United States Economy, caps. 2-4 y apéndice, Nueva York, Macmillan, 1991.

, "The Rate of Surplus-value in the United States: 1947-77”, disertación, Boston, University of Massachusetts, 1983.

Ricardo, David, Principios de economía política y tributación, caps. II, VI, XXI, XXIV, XXVI, México, FCE, 1982.

Shaikh, Anwar y M. Tonak, Measuring the Wealth of Nations, Cambridge, Cambridge University Press, 1994.

Sharpe, Donald Andrew, "The Structure of the Canadian Economy, 1961-1976: A Marxian Input-output Analysis", disertación, McGill University, 1982.

Sistema de Cuentas Nacionales de México, Banco de información económica, 2004, en http:// www.inegi.gob.mx.

Smith, Adam, Investigación sobre la riqueza y causas de la riqueza de las naciones, Libro primero, caps. IX y XI; libro segundo, cap. III, México, FCE, 1958.

Smith Murray, E.G., "Productivity, Valorization and Crisis: Socially Necessary Unproductive Labor in Contermporary Capitalism", en Science and Society, vol. 57, núm. 3, otoño de 1993, pp. 162-293.

Valle Baeza, Alejandro, Economía Política II: Teoría del valor, Curso básico de Economía (versión preliminar), Facultad de Economía. UNAM, México, inédito, 2005. 